\title{
LAMP Method as One of the Best Candidates for Replacing with PCR Method
}

\author{
Masoud KEIKHA
}

Submitted: 1 Aug 2017

Department of Microbiology, School of Medicine, Isfahan Medical University, Accepted: 1 Oct 2017

Isfahan, Iran

Online: 28 Feb 2018

To cite this article: Keikha M. LAMP method as one of the best candidates for replacing with PCR method. Malays J Med Sci. 2018;25(1):121-123. https://doi.org/10.21315/mjms2018.25.1.15

To link to this article: https://doi.org/10.21315/mjms2018.25.1.15

\section{Dear Editor,}

Molecular methods have been recently developed in studies; one of the most applicable molecular methods is polymerase chain reaction (PCR). PCR, which has attracted the attention of the biologists, is a fast and sensitive method capable of amplifying small numbers of target DNA copies across millions orders of magnitude. Due to its high efficiency and power, this method has been widely applied in medical microbiology. In spite of its advantages, this method has also some limitations such as use of thermal cycles for multiplication (30-40 cycles), use of expensive tools such as thermocycler, detection methods of PCR product which always suffer from problems like use of toxic materials such as Ethidium bromide (which is carcinogenic material). Such limitations have caused to the condition that this method can be only applied in wellequipped laboratories benefiting from highly experienced staff. Therefore, this method can't be employed in places lacking well-equipped labs $(1,2)$. In this regard, development of a simpler method with higher efficiency which could be also implemented in ordinary labs even by nonexperienced people is needed.

One of the main molecular detection methods in which the multiplication is carried out in isothermal condition and does not require thermocycler is Loop Mediated Isothermal Amplification (LAMP) designed in 2000 by Notomi et al. (14). In this technique, 6 highly selective primers named internal primers [Forward Inner Primer (FIP) (Forward 1 Complementary (F1C), F2) \& Backward Inner Primer (BIP) (Backward 1 Complementary (B1C), B2)], external primers (F3, B3) and loop- specific primers [Forward Loop Primer (FLP), Backward Loop Primer (BLP)] are used. Internal primer of FIP in DNA pattern will attach to F2complementing region in the pattern strand and initiate the synthesis of the complementing strand. By location of F3 and B3 external primers with the help of DNA polymerase enzyme of Bst (Bacillus stearothermophilus), the strand length would be elongated which will lead to generation of a dumbbell-shaped DNA structure. This stem-loop structure of DNA will act as the LAMP initiator. FIP will attach to stem-loop structure of DNA and initiate the synthesis of successor strand which will generate a stem-loop intermitted DNA structure possessing an inverse copy of target sequence in its stem-loop region produced in the other end of the gen by BIP primer. The released strand forms a structure with external loop which acts as the pattern for BIP polymer. Finally dumbbell-shape DNA is produced which will act as the step material of LAMP cycle. By design of oligonucleotide probes specific for these structures, they can be applied in hybridisation; therefore there will be no need for heat denaturation after the multiplication. This means that all the steps from multiplication to detection could be carried out in the same temperature. The final product is stem-loop DNAs with several inverse sequences of the target DNA having a cauliflower-like structure with multiple loops $(3-5)$. The molecular basis of LAMP is based on turbidity due to the production of Magnesium pyrophosphate $\left(\mathrm{Mg}_{2} \mathrm{P}_{2} \mathrm{O}_{7}\right)$; while in recently years, utilisation of SYBR Green dye has made comfortable this step (6). 
The Loop Mediated Isothermal Amplification method has been used to diagnosis of various infectious disease, identification and differentiation of pathogenic microorganisms; for example Mycobacterium tuberculosis, Nocardia spp., Pseudomonas fluorescens, Staphylococcus aureus, Helicobacter pylori, Salmonella species and several other bacteria and medically important viruses (7-13).

Advantages of this method includes: (i) capability of LAMP technique in multiplication in isothermal condition (between $59{ }^{\circ} \mathrm{C}-66{ }^{\circ} \mathrm{C}$ ) and it can start the detection with small number of DNA, (ii) LAMP provides accessible, costeffective, easy-to-perform method, (iii) reaction products are mixture of hairpin structure DNAs with different sizes and cauliflower-like structure having several loops formed from annealing between repetitive inverse sequences of the target sequence which can be easily observed under optical microscopes facilitating the possibility to selective detection, (iv) LAMP technique is highly specific as its can detect 6 gene regions of the target sequence by its 4 primers at the beginning of the reaction, and 4 also other regions during the LAMP reaction procedure, (v) LAMP is a simple method only needs primer, DNA polymerase and reaction mixture; it does not need thermocycler and can be conducted in warm water bath or thermal blocks, (vi) the created turbidity due to gene multiplication and magnesium pyrophosphate $\left(\mathrm{Mg}_{2} \mathrm{P}_{2} \mathrm{O}_{7}\right)$ precipitants in reaction medium does not need electrophose-gel for detection and identification of reaction product; RNA sequences can be also multiplied by this method and inverse copying $(7-8,14)$. However, this technique has some limitations. Complexity of multiple primers' designs for multiplication of new gene regions and selecting the suitable regions in the gene sequence for efficient design of primers, complicated product having cauliflower-like structures with different sizes, lack of commercial kits based on LAMP technique and complexity of this method mechanism are some of its drawbacks limiting its popularity among the researchers; and it not as common as PCR-based method. But LAMP method has significant advantages which by some modifications could be an important alternative for PCR method.

\section{Conflict of interest}

There is no conflict of interest.

\section{Correspondence}

Dr Masoud Keikha

MSc Microbiology (Isfahan Medical University, Iran)

Department of Microbiology, School of Medicine,

Isfahan Medical University, Isfahan, Iran.

Tel: +989386836425

E-mail: masoud.keykha9o@gmail.com

\section{References}

1. Kumar A, Arora V, Bashamboo A, Ali S. Detection of Salmonella typhi by polymerase chain reaction: implications in diagnosis of typhoid fever. Infect Genet Evol. 2002;2(2):107-110.

2. Hoorfar J, Ahrens P, Rådström P. Automated $5^{\prime}$ nuclease PCR assay for identification of Salmonella enterica. $J$ Clin Microbiol. 2000;38(9):3429-3435.

3. Nagamine K, Hase T, Notomi T. Accelerated reaction by loop-mediated isothermal amplification using loop primers. Mol Cell Probe. 2002;16(3):223-229.

4. Nagamine K, Watanabe K, Ohtsuka K, Hase T, Notomi T. Loop-mediated isothermal amplification reaction using a nondenatured template. Clin Chem. 2001;47(9):1742-1743.

5. Mori Y, Hirano T, Notomi T. Sequence specific visual detection of LAMP reactions by addition of cationic polymers. BMC Biotechnol. 2006;6(1):3.

6. Rivero R, Bisio M, Velázquez EB, Esteva MI, Scollo K, González NL, et al. Rapid detection of Trypanosoma cruzi by colorimetric loopmediated isothermal amplification (LAMP): a potential novel tool for the detection of congenital Chagas infection. Diagn Microbiol Infect Dis. 2017;89(1):26-28.

7. Kawano S, Maeda T, Watanabe J, Fujikura Y, Mikita K, Hara Y, et al. Successful diagnosis of tuberculous lymphadenitis by loop-mediated isothermal amplification of cutaneous samples from an ulcerated surface lesion: a case report. J Med Case Rep. 2014;8(1):254. 
8. Itano $\mathrm{T}$, Kawakami $\mathrm{H}$, Kono $\mathrm{T}$, Sakai $\mathrm{M}$. Detection of fish nocardiosis by loop-mediated isothermal amplification. $J$ Appl Microbiol. 2006;100(6):1381-1387.

9. Saharan P, Duhan JS, Gahlawat SK. Detection of Pseudomonas fluorescens from broth, water and infected tissues by loop-mediated isothermal amplification (LAMP) method. Afr J Biotechnol. 2015;14(14):1181-1185.

10. Su J, Liu X, Cui H, Li Y, Chen D, Li Y, et al. Rapid and simple detection of methicillin-resistance Staphylococcus aureus by orfX loop-mediated isothermal amplification assay. BMC Biotechnol. 2014;14(1):1-8.

11. Bakhtiari S, Alvandi A, Pajavand $H$, Navabi J, Najafi F, Abiri R, et al. Development and diagnostic evaluation of loop-mediated isothermal amplification using a new gene target for rapid detection of Helicobacter pylori. Jundishapur $J$ Microbiol. 2016;9(5):e28831.
12. Kokkinos P, Ziros P, Bellou M, Vantarakis A. Loop-mediated isothermal amplification (LAMP) for the detection of Salmonella in food. Food Anal Methods. 2014;7(2):512-526.

13. Wang J, Lu P, Yan J, Zhang Y, Huang L, Ali Z, et al. Rapid and sensitive detection of RNA viruses based on reverse transcription loop-Mediated isothermal amplification, magnetic nanoparticles, and chemiluminescence. J Biomed Nanotechnol. 2016;12(4):710-716.

14. Notomi T, Okayama H, Masubuchi H, Yonekawa $\mathrm{T}$, Watanabe K, Amino N, et al. Loop-mediated isothermal amplification of DNA. Nuc Acids Res. 2000;28(12):63-70. 